\title{
La tesis de la brutalización (George L. Mosse) y sus críticos: un debate historiográfico ${ }^{1}$ \\ The 'Brutalization' Thesis (George L. Mosse) and its Critics: A Historiographical Debate
}

\author{
Ángel Alcalde \\ Instituto Universitario Europeo, Florencia \\ angel.alcalde@eui.eu
}

Recibido: 31-I-2016

Aceptado: 5-IX-2016

\section{Resumen}

Este artículo explora el amplio debate internacional mantenido por historiadores contemporaneístas en torno a la tesis de la "brutalización", popularizada por George L. Mosse a partir de su libro Soldados caídos (1990), y según la cual la experiencia de guerra de los soldados del frente en la Primera Guerra Mundial habría sido la causa de los altos niveles de violencia política de la República de Weimar y, por ende, el origen del nacionalsocialismo y el genocidio. El artículo clarifica las raíces de la interpretación mosseana, analiza las críticas, reformulaciones y usos de la "brutalización" y concluye que el fuerte debate mantenido, a pesar de no alcanzar consenso, terminó por consagrar una noción sugerente, aunque obscura y de cuestionable capacidad analítica, en el lenguaje profesional de los historiadores.

Palabras clave: Brutalización, Historiografía, George L. Mosse, Violencia política, Entreguerras, Primera Guerra Mundial.

1. El autor agradece a Jesús Casquete y a los organizadores y participantes del seminario HISTAGRA (Universidade de Santiago de Compostela), donde se presentó una versión anterior del texto, sus críticas y comentarios. Este trabajo ha sido posible gracias al apoyo del programa "Salvador de Madariaga", financiado por el Ministerio de Educación. El autor ha formado parte del proyecto "Discursos e identidades de género en las culturas políticas de la derecha española, 1875-1975”, HAR2012-32539. 


\begin{abstract}
This article explores the widespread international debate in modern historiography maintained around the "brutalization" thesis, which was popularized in George L. Mosse's book Fallen Soldiers (1990). According to Mosse, the war experience of the front soldiers during World War I was the cause of the heightened levels of political violence during the Weimar Republic. Such brutalization allegedly provided the basis for Nazism and the Genocide. In an attempt to clarify the origins of Mosse's interpretation this work analyses criticism, reformulations and uses of "brutalization". In spite of the fact that the heated debate reached no consensus, it eventually managed to establish a revealing -though vague and scarcely open to analytical potential notion in the professional language of historians.
\end{abstract}

Keywords: Brutalization, Historiography, George L. Mosse, Political Violence, Interwar Period, World War I.

En 1936, un adolescente judío alemán, de 17 años, llamado George Lachmann Mosse, viajó con su madre en visita turística a Italia. Por entonces, años del "consenso" bajo el régimen fascista de Mussolini, y de exaltación ultranacionalista alemana bajo Hitler, una familia judía muy influyente como eran los Mosse todavía podía moverse con agilidad, si no sin miedo, por la mayoría de países europeos, aunque sus miembros ya habían abandonado Alemania, para instalarse sucesivamente en Suiza, Francia e Inglaterra. En sus borrosos recuerdos de aquel viaje al país transalpino, Mosse describe haber visitado la ciudad de Florencia, donde "presenció lo que hoy se llamaría un ejercicio de participación popular" ("witnessing what today would be called an exercise of popular participation"): ${ }^{2}$

A division, or a regiment perhaps -I do not remember which- had returned to Florence from fighting in the Ethiopian war. How were they welcomed? The soldiers were allowed to take over the city: they regulated the traffic and in their bright uniforms even drove streetcars. These very young men were rewarded by being given the chance to participate in actions which have always been tempting to youth (I myself remember how I would have liked to regulate traffic and drive a streetcar). ${ }^{3}$

Esta experiencia serviría para confirmar al joven Mosse, futuro historiador, sus tempranas intuiciones sobre el poder de las ideologías fascista y nacional-socialista para nacionalizar a las masas a través de la irracionalidad, el mito y la vivencia de comunidad en un marco participativo y de entusiasmo revolucionario.

Muchas décadas después, y más de diez años tras la muerte de Mosse, cuando sus sugerentes tesis, argumentos y explicaciones sobre la cultura y la ideo-

2. MOSSE, George L., Confronting History. A memoir, Madison, University of Wisconsin, 2000, p. 108.

3. Ibid., p. 109. 
logía nazi y fascista se han consolidado en la historiografía, un historiador puede intentar en vano documentar históricamente esa anécdota que plasmó en sus memorias. En el verano de 1936, en efecto, el retorno de las tropas italianas de su expedición a Etiopía se realizó entre grandes ceremonias de bienvenida, en los puertos de Nápoles o Livorno, alrededor de las estaciones ferroviarias de llegada en Roma, Florencia o Milán. Normalmente, la población reunida por las autoridades les ofrecía un gran recibimiento, con banderines, orquestas, cánticos de Giovinezza. Las unidades militares, con sus uniformes y cascos coloniales, desfilaban entre el alborozo de la estación a los cuarteles, donde permanecían hasta la definitiva desmovilización de sus soldados. En ocasiones, los ayuntamientos ofrecían recepciones y brindis a los oficiales o excombatientes locales, que en verdad gozarían de una serie de beneficios materiales, ganados por su servicio en las tierras del Imperio, aunque a medio plazo también el desempleo y la desorientación les afectarían. Todo esto se comprueba documentalmente para el caso de la ciudad de Florencia, visitada por Mosse en 1936, y en particular para la acogida que se dio en julio de ese año a la división Gavinana. ${ }^{4}$

Sin embargo, a pesar de una búsqueda específica al respecto, ${ }^{5}$ es imposible documentar que allí se recompensara a los veteranos permitiéndoles "tomar el control de la ciudad", regulando el tráfico con sus "brillantes uniformes" o conduciendo los tranvías. Pero tampoco esta descripción carece de toda base. En realidad, una de las medidas que la ciudad de Florencia, a la altura de 1936, había concedido a la fascistizada Associazione Nazionale Combattenti (que en la provincia contaba con más de 17.000 miembros), fue asignar a sus veteranos de guerra las tareas de vigilancia de los aparcamientos de bicicletas del centro, y de los vehículos de reparto de fruta y verdura del Mercado de Sant'Ambrogio. ${ }^{6}$ En cuanto al tráfico de la ciudad toscana, se sabe que en esta década empeoró notablemente, en parte a causa del gran aumento de vendedores ambulantes, muchos de ellos excombatientes de la Gran Guerra a los que el Comune no se atrevió a rechazar su petición de licencia. En los años 30 fue necesario aumentar el número de guardias de circulación de 263 a 349, que vestían un nuevo uniforme más elegante, con porra, y que debían realizar el saludo fascista cuando montaban y desmontaban el turno. ${ }^{7}$ Se diría que con todos estos mimbres

4. La Nazione (Florencia), 9, 10, 13-15, 22-24 y 30-VII-1936.

5. A través de la consulta de la prensa diaria florentina y de los fondos del Archivio Storico del Comune di Firenze.

6. Associazione Nazionale Combattenti. Federazione Provinciale di Firenze, Relazione Morale e Finanziaria. Ottobre XIV, Florencia, s. f. [1935], pp. 25-30.

7. PIERACCINI, Paolo, Il Corpo di Polizia Municipale di Firenze. Dai Lorena all'Itaia repubblicana, Florencia, Paganini, 2004, pp. 154-177, 218-227, y una fotografía del uniforme en p. 166. 
probablemente percibidos en su visita, Mosse construyó su recuerdo, adornándolo y creando una imagen casi mágica de un régimen fascista que recompensa a sus excombatientes poniendo en sus manos el control del tráfico de la ciudad, como él recogió en sus memorias.

Sea como fuere, esta anécdota, y nuestra contraposición de la idea mental -muy humana- transmitida por Mosse frente su realidad histórica -documentada-, creemos que sirven para plasmar una contradicción clave existente en el debate historiográfico sobre la idea mosseana de "brutalización" que examinamos en este artículo. Esta es: el choque entre una visión de la historia fuertemente cultural, subjetivista, interpretativa, reveladora de fuerzas históricas casi intangibles y ocultas que supuestamente marcaron la larga evolución de las sociedades europeas en la modernidad (esto es, la manera de George L. Mosse de entender la historia), y por otro lado la necesidad, en el oficio científico del historiador, de analizar la realidad a partir de las fuentes documentales, con una mínima aspiración a la objetividad y al conocimiento contrastable, falsable, que permita una conceptualización amarrada a lo empíricamente perceptible.

Con el análisis del debate internacional, o en cierto sentido transnacional, mantenido durante muchos años en torno a la tesis de la "brutalización" no sólo pretendemos, pues, conocer mejor la aportación de un historiador clave para la historia del siglo XX, sino revelar diversas maneras en que los conceptos historiográficos se transfieren, comprenden, circulan y aplican en diversos contextos y momentos intelectuales, y con qué resultados.

\section{El autor y su obra}

En la inmensa obra historiográfica de George L. Mosse (1918-1999), ${ }^{8}$ la tesis de la brutalización representa solamente una pequeña, aunque influyente parte. Mosse, que desarrolló su larga carrera en los Estados Unidos, como profesor en la Universidad de Wisconsin desde 1955, se destacó primero como modernista, escribiendo obras de éxito sobre esa época durante los años 50. Fue a partir de los años 60 cuando, tras publicar una obra muy sugerente y de profunda mirada sobre los siglos XIX y XX como fue The Culture of Western Europe (1961), su labor dio un giro que le llevaría a convertirse en un estudioso de las raíces culturales del nazismo. Durante varias décadas, The Crisis of German Ideology

8. Existen numerosas aproximaciones a la ingente obra de Mosse, y una solvente biografía intelectual: PLESSINI, Karel, The Perils of Normalcy. George L. Mosse and the Remaking of Cultural History, Madison, University of Wisconsin Press, 2014. Por su concisión resulta útil ASCHHEIM, Steven E., "George Mosse at 80: A Critical Laudatio", Journal of Contemporary History, vol. 34, no 2 (1999), pp. 295-312. 
(1964) fue su libro más importante, pues con su examen de las raíces ideológicas del nacional-socialismo alemán y la evolución del nacionalismo völkisch, presentada a la manera de un Sonderweg germano, Mosse se alejaba de la entonces dominante interpretación del nazismo como totalitarismo. A este volumen siguieron otros, como la selección de textos sobre la ideología nazi bajo el entonces atrevido título Nazi Culture (1966), traducido a varios idiomas, y una serie de artículos y obras colectivas e individuales en la misma línea investigadora. La obra de Mosse pasó de enmarcarse en el campo de la historia de las ideas, a abrazar el estudio de la ideología como elemento cultural para la construcción del consenso político, y de ahí al análisis de los mitos, símbolos y estereotipos visuales en la sociedad moderna.

Como adelantado a su tiempo, su manera de interesarse por la cultura nazi resultó sumamente provocadora, y exitosa, ya que no tardarían en aparecer émulos en otras historiografías, en particular en la italiana, especialmente durante los años 80. Se sabe que The Crisis of German Ideology, traducida al italiano ya en 1968, fue una lectura que causó impacto en Renzo De Felice, inmerso entonces en la escritura de su conocida biografía de Mussolini, y que asimismo influyó muy notablemente a un discípulo de aquel, Emilio Gentile, que por su parte preparaba su conocida tesis sobre la ideología fascista. ${ }^{9}$ No en vano, Gentile y otros historiadores de renombre como Roger Griffin no han cesado de ensalzar al historiador de origen judeo-alemán, por haber desbrozado el camino a las renovadoras perspectivas culturalistas sobre el fascismo que tanto se han cultivado en las últimas décadas. ${ }^{10}$

Aunque Mosse ha pasado a la historia de la historiografía, sobre todo, como un especialista en el nazismo, sus perspectivas siempre llegaron muchísimo más lejos en el pasado, abordando temáticas que trascendían ampliamente la cultura política alemana de los años 30. Los años de mayor actividad profesional y producción intelectual de Mosse se sitúan entre mediados de los años 60, cuando fundó junto a Walter Lacqueur el Journal of Contemporary History (1966), y finales de los años 80. En este tiempo, otra obra de enorme relevancia fue The Nationalization of the Masses (1975), un estudio de los símbolos, mitos y liturgias del culto a la nación en Alemania, con el que Mosse también se con-

9. ARAMINI, Donatello, George L. Mosse, L'Italia e gli storici, Franco Angeli, Milano, 2010.

10. GENTILE, Emilio, Il fascino del persecutore. George L. Mosse e la catastrofe dell'uomo moderno, Roma, Carocci, 2007; GRIFFIN, Roger, "Fascism and Culture: A Mosse-Centric Meta-Narrative (or how Fascist Studies Reinvented the Wheel)", en António COSTAPINTO, ed., Rethinking the Nature of Fascism. Comparative Perspectives, Basingstoke, Palgrave Macmillan, 2011, pp. 85-116. 
sagró como historiador del nacionalismo. ${ }^{11} \mathrm{Al}$ igual que con sus trabajos sobre la historia del racismo, que en 1978 dieron como fruto la obra Toward the Final Solution, lo que Mosse pretendía explicar era, ante todo, las fuentes ideológicas y culturales del nazismo, de cuya persecución él mismo había tenido que escapar por su condición de judío. Además, su homosexualidad inconfesa durante mucho tiempo, que le había convertido en un "doble outsider", también le empujó a innovar en el estudio de la masculinidad y la sexualidad, en unos años en los que la historia de género estaba todavía en ciernes.

\section{Hacia la tesis de la brutalización}

Como ocurrió con otras de sus obras, la publicación de Soldados caídos en $1990,{ }^{12}$ libro en el que se desgrana la tesis mosseana de la "brutalización", estuvo precedida por una retahíla de contribuciones menores, normalmente en forma de artículos, en las que el autor dejó constancia de las numerosas ideas e interpretaciones que sintetizaría el volumen definitivo publicado por Oxford University Press. Así, desde finales de los años 70, Mosse leyó y escribió acerca de temas como la muerte y el enterramiento, el culto a los caídos, ${ }^{13}$ la cultura de la Primera Guerra Mundial y, sobre todo, lo que él llamó el Mito de la Experiencia de Guerra. ${ }^{14}$ Éste era el concepto clave que vehicularía todo el libro. Se trataba de una narrativa mítica sobre la experiencia bélica que incorporaba varios temas: el espíritu de 1914, la guerra como prueba de virilidad, el ideal de camaradería y el culto al soldado caído. Como Mosse explicaría en Fallen Soldiers, el Mito implicó también una apropiación de la naturale$z a$, visible incluso en el diseño de los cementerios de guerra; el éxito de la mitificación durante el periodo de entreguerras -cuando fue clave en el ascenso de la extrema derecha sobre todo el Alemania- se debió a los procesos de "trivialización" de la guerra y de la "brutalización" producida por su experiencia.

11. Hay traducción en español: MOSSE, George L., La nacionalización de las masas. Simbolismo político y movimientos de masas en Alemania desde las guerras napoleónicas al Tercer Reich, Madrid, Marcial Pons, 2005; sobre el tardío impacto de esta obra en España véase CASPISTEGUI, Francisco Javier, "La nacionalización de las masas y la historia del nacionalismo español", Ayer, no 94 (2014), pp. 257-270. La traducción al italiano no se hizo esperar tanto (1975, Il Mulino).

12. MOSSE, George L., Fallen Soldiers. Reshaping the Memory of the World Wars, New York, Oxford University Press, 1990. Traducido al español por Ángel Alcalde: MOSSE, George L., Soldados caídos. La transformación de la memoria de las guerras mundiales, Zaragoza, Prensas de la Universidad de Zaragoza, 2016.

13. MOSSE, George L., "National Cemeteries and National Revival: The Cult of the Fallen Soldiers in Germany", Journal of Contemporary History, vol. 14, no 1 (Jan. 1979), pp. 1-20.

14. MOSSE, George L. "Two World Wars and the Myth of the War Experience", Journal of Contemporary History, vol. 21, nº 4 (1986), pp. 491-513. 
Dejando al margen diversos temas y conceptos que Mosse incorporó a su libro, nos centraremos aquí solamente en la tesis de la "brutalización".

La propia palabra requiere una exploración previa. Como puede comprobarse fácilmente a través de la búsqueda de texto en hemerotecas digitales, no se trata de un neologismo, ya que puede documentarse su uso en la lengua inglesa (brutalization) y en la francesa (brutalisation), en el mismo significado semántico que le atribuía Mosse, ya durante el siglo XIX. En 1892, por ejemplo, el artículo de un diario de noticias publicado en Indianápolis (Estados Unidos) mencionaba de pasada el "efecto brutalizador de la guerra sin sentido", al hablar de cómo los sistemas penales producían una "brutalización de los hombres y mujeres". ${ }^{15}$ Aunque no era una palabra de uso común, probablemente se utilizó más habitualmente a partir de la Primera Guerra Mundial para expresar, entre otras cosas, la existente preocupación de que los excombatientes que retornaban a los hogares se hubiesen convertido en individuos violentos y conflictivos. Significativamente, en 1922, una revista publicada por un grupo de mutilados de guerra franceses afirmaba, al comentar los efectos de la guerra sobre el sistema nervioso de millones de hombres expuestos al combate, que "la 'brutalización' de las masas, después de cada guerra, siempre se ha constatado". ${ }^{16}$ En alemán, la voz Brutalisierung también aparece utilizada con asiduidad ya en el XIX y durante la Gran Guerra, aunque predominase su otro sentido semántico: como sinónimo de agresión o violencia ejercida contra algo o alguien (un significado algo diferente que era también más común en la lengua francesa). Con esta presencia léxica, no extraña que en la Italia de entreguerras también llegara a utilizarse el vocablo brutalizzazio$n e:$ es interesante mencionar que una reseña italiana, muy crítica, de la obra de Remarque Sin Novedad en el Frente (1929), lamentaba que su supuesto intento de humanizar la guerra resultaba en cambio en "brutalización y bestialización". ${ }^{17}$ En español, por aquellas fechas la palabra probablemente sólo se utilizaba al traducirse del inglés o francés, y así encontramos que una revista publicada en 1923 la transmitía en unas declaraciones del estadista británico Lord Lansdowne describiendo los efectos de la pasada guerra, que no eran sino "la ruina de los fundamentos de la sociedad, la brutalización del carácter humano, la liberación de las pasiones $[\ldots]$ ", etc. ${ }^{18}$

15. ("brutalizing effect of needless warfare", "brutalization of men and women"), The Indiana Sentinel (Indianapolis), 21-II-1892.

16. ("La 'brutalisation' des masses, après chaque guerre, à toujours été constatée"), Le Mutilé de l'Algerie. Journal des mutilés, reformés et blessés de guerre de l'Afrique du Nord (Argel), $\mathrm{n}^{\circ} 258,18$-VIII-1922.

17. ("diventa brutalizzazione e imbestiamento"), La Stampa (Turín), 11-V-1929.

18. La Velocidad. Órgano de la sociedad de chauffeurs y aspirantes (Madrid), 1 de febrero de 1923. 
En definitiva, existía ya en la primera posguerra mundial un miedo a los efectos negativos de la experiencia bélica sobre la personalidad de los individuos, en particular a través de la exacerbación de los instintos agresivos, y ocasionalmente se empleaba la palabra "brutalización" para definir esta secuela, que podía extenderse, se decía, al conjunto de la sociedad: ${ }^{19}$ la tesis mosseana de la "brutalización", en pocas palabras. Esto no significa que el concepto calara también en el lenguaje científico o en el historiográfico, cosa que no ocurrió, aparentemente, hasta mucho más tarde.

En los años 70, sociólogos norteamericanos propusieron la hipótesis del "efecto brutalizador" que la pena de muerte podía ejercer sobre la sociedad. En particular, algunos investigadores trataron de evaluar el impacto de este castigo, a través del número de homicidios cometidos con posterioridad a las ejecuciones, cuando éstas habían sido dadas a conocer a través de la prensa. Lo que sugerían era que la publicidad sobre la pena capital provocaba "brutalización", es decir, más crímenes, y no disuasión. Nunca ha podido establecerse, no obstante, una clara relación de causa-efecto entre la divulgación de las ejecuciones de reos y la comisión de más -no menos- asesinatos en los estados donde se llevan a cabo, pero ese debate científico fue suficientemente relevante como para pasar a ser de dominio público en los años 70 y $80 .{ }^{20}$ Muy probablemente, este significado sociológico del concepto de "brutalización" influyó en Mosse para dar forma a su propia tesis histórica en esas décadas.

Aunque la tesis de la "brutalización" mosseana no surgiera de la nada, como vemos, no dejaba de ser sumamente original su aplicación a la historia cultural de la Primera Guerra Mundial y del periodo de entreguerras. Pero también de estos campos de estudio se nutrió: desde finales de los años 70 y hasta finales de los 80 se publicaron una serie de obras clave que abordaron la Gran Guerra desde innovadoras perspectivas culturalistas y que estudiaron temáticas relativamente nuevas como los movimientos excombatientes y la experiencia de combate. En 1975 se publicó el influyente libro de Paul Fussell, The Great War and Modern Memory, ${ }^{21}$ que valorizó la literatura de guerra como

19. Véase LAWRENCE, Jon, "Forging a Peaceable Kingdom: War, Violence, and Fear of Brutalization in Post-First World War Britain", The Journal of Modern History, vol. 75, $n^{\circ} 3$ (2003), pp. 557-589.

20. KING, David R., "The Brutalization Effect: Execution Publicity and the Incidence of Homicide in South Carolina", Social Forces, vol. 57, n 2 (1978), pp. 683-687; BAILEY, William C., "Deterrence, Brutalization, and the Death Penalty: Another Examination of Oklahoma's Return to Capital Punishment", Criminology, vol. 36, nº 4 (1998), pp. 711-734.

21. FUSSELL, Paul, The Great War and Modern Memory, Oxford, Oxford University Press, 1975. Hay traducción al español: Id., La Gran guerra y la memoria moderna, Madrid, Turner, 2006. 
fuente para entender la gran transformación de la mentalidad y el lenguaje producida por la experiencia bélica, al tiempo que la obra dirigida por Stephen R. Ward, The War Generation, ${ }^{22}$ reunía varios estudios sobre los movimientos excombatientes de varios países beligerantes. $\mathrm{Al}$ año siguiente apareció la del historiador militar John Keegan, The Face of Battle, ${ }^{23}$ que describía la vivencia de combatir de una manera profunda, realista y desde el punto de vista del soldado, para el cual, argumentó el autor, "la batalla tiene lugar en un ambiente salvajemente inestable tanto física como emocionalmente" ${ }^{24} \mathrm{Al}$ impacto causado por la experiencia de guerra sobre la identidad de los combatientes dedicó el historiador Eric J. Leed un fascinante estudio de corte históricoantropológico en 1979, No Man's Land, en el que se describía el traumático "rito de paso" atravesado por quienes lucharon en las trincheras del frente occidental. ${ }^{25}$ Ese mismo año, Robert Wohl hablaba de aquella generación de excombatientes, "portadores de una revelación que podría revolucionar la vida civil". ${ }^{26}$

Mosse, que por aquellas fechas estaba ya inmerso en su propio estudio del proceso de glorificación de la experiencia de guerra en diversos países, en el cual organizaciones de excombatientes de diverso signo político habrían jugado un rol crucial, ${ }^{27}$ ya utilizaba a la altura de 1977 la noción de "brutalización" para explicar la fuerte presencia de la violencia política en el periodo de entreguerras. En la entrevista sobre el nazismo que mantuvo con el historiador norteamericano Michael A. Ledeen y que se publicó primeramente en italiano, Mosse explicó lo que el llamaba la "brutalización de la vida" sucedida tras la Primera Guerra Mundial en Alemania, y también en Italia:

La única novedad traída por la guerra a toda Europa fue una brutalización de la vida. Esta fue el producto de la glorificación de fenómenos de muerte de masas de una dimensión nunca vista en el pasado [...]. Todo aquello condujo a una suerte

22. WARD, Stephen R., ed., The War Generation. Veterans of the First World War, London y Port Washington (NY), Kennikat Press, 1975.

23. KEEGAN, John, The Face of Battle, London, Jonathan Cape, 1976.

24. Citamos según la traducción española, KEEGAN, John, El rostro de la batalla, Madrid, Ediciones Ejército, 1990, p. 58.

25. LEED, Eric J., No Man's Land. Combat and Identity in World War I, Cambridge, Cambridge University Press, 1979.

26. ("bearers of a revelation that might revolutionize civilian life") WOHL, Robert, The Generation of 1914, Cambridge, Ma., Harvard University Press, 1979, p. 52.

27. MOSSE, George L., "La sinistra europea e l'esperienza della guerra (Germania e Francia)", en Rivoluzione e Reazione in Europa 1917/1924. Convegno storico internazionale - Perugia 1978, Roma, Mondo Operaio - Edizioni Avanti!, 1978, vol. II, pp. 151-167. En esta publicación, Mosse ya cita las principales obras existentes sobre movimientos excombatientes del periodo de entreguerras. 
de glorificación de la brutalidad bajo el signo de la lucha, glorificación que en Alemania se prolongó durante el periodo posbélico. [...] En definitiva, la brutalización generada por la guerra se prolongó en el periodo posbélico; y lo mismo, creo, sucedió en Italia. Y este fue el terreno, en cierto sentido, del que se nutrió el fascismo, traduciendo la brutalización en una suerte de activismo y combinando por tanto este último no sólo con el culto a los héroes, el culto a los caídos, sino también con la moralidad burguesa, la ideología de la ley y orden, etc. ${ }^{28}$

De este modo, la noción de brutalización entró con fuerza en el lenguaje académico, y en particular en los estudios acerca de la violencia del periodo posbélico, en los que las acciones del Freikorps y las obras literarias militaristas y violentas de sus miembros Ernst Jünger y Ernst von Salomon se citaban asiduamente. Incluso el intelectual Norbert Elias, que contrapuso la persistencia de la violencia posbélica a su idea del proceso de civilización, habló del ethos bélico germano representado por aquellos paramilitares, protagonistas de una "brutalización" que "romantizaba y glorificaba la brutalidad", ${ }^{29}$ y que pasaron, se decía, casi en bloque a las filas de Hitler en los años 30. ${ }^{30}$ Otra obra influyente en la historia cultural de la Gran Guerra, la de Modris Eksteins, Rites of Spring (1989), ${ }^{31}$ también incidiría en esa visión de violentos excombatientes como heraldos del fascismo. En general, los años 80 presenciaron la eclosión de la historia cultural de la Primera Guerra Mundial, con gran fuerza en la historiografía italiana, en la que Mosse ejerció un enorme influjo. ${ }^{32} \mathrm{Su}$ tesis de la brutalización de la política alemana, ya muy desarrollada en una contribución de 1987, empezaba a abrirse paso. Según su autor, la brusca confrontación con la muerte durante la guerra había "sin duda transformado la actitud de los soldados hacia la vida", haciéndoles indiferentes ante la muerte, aunque no podía saberse cómo esa indiferencia había contribuido a la "apro-

28. Traducción mía de MOSSE, George L. y Michael A. LEDEEN, eds., Intervista sul nazismo, Roma-Bari, Laterza, 1977, pp. 44-45. La versión inglesa se publicó más tarde: MOSSE, George L., Nazism. A Historical and Comparative Analysis of National Socialism, Oxford, Basil Blackwell, 1978.

29. ELIAS, Norbert, "Civilización y violencia", Reis, no 65 (1994), pp. 141-151, cita en p. 149; traducción al español de un texto alemán publicado en 1981 a partir de una conferencia de Elias pronunciada en 1980.

30. Esta interpretación sobre el Freikorps fue popularizada por JONES, Nigel H., Hitler's Heralds. The Story of the Freikorps, 1918-1923, London, Murray, 1987.

31. EKSTEINS, Modris, Rites of Spring. The Great War and the Birth of the Modern Age, Boston, Houghton Mifflin Company, 1989. Recientemente traducido al español: Id., La consagración de la primavera. La Gran Guerra y el nacimiento de los tiempos modernos, Valencia, Pre-Textos, 2014.

32. ARAMINI, Donatello, George L. Mosse..., pp. 141-145; véase LEONI, Diego y Camillo ZADRA (eds.), La Grande Guerra: Esperienza, memoria, immagini, Bologna, Il Mulino, 1986. 
bación del tono brutal de la política posbélica y luego a la aceptación de la política nazi". ${ }^{33}$

Finalmente, en 1990 se publicó Fallen Soldiers, aclamado libro que había tenido un largo proceso de gestación, y en el que se desplegaba plenamente, en uno de sus capítulos, la tesis de la brutalización. Entendida como una consecuencia del encuentro de los soldados con la muerte de masas en la Primera Guerra Mundial; la brutalización ocurrió, según escribió un reseñista, "no simplemente porque la gente se hizo brutal, sino también porque aprendió a ver lo brutal como algo bello". ${ }^{34} \mathrm{Al}$ margen de que esta tesis provocara un cierto debate historiográfico, cabe destacar que Fallen Soldiers fue un inmediato éxito internacional; su traducción italiana apareció prácticamente al mismo tiempo que la inglesa: ${ }^{35}$ la alemana lo hizo en $1993 ;{ }^{36}$ la israelí, en 1994:37 las reseñas positivas, no sólo en los medios especializados sino también en la prensa, fueron numerosas ${ }^{38}$ e incluso se realizó, al parecer, un documental televisivo sobre el contenido de la obra, grabado en diversas partes de Europa..$^{39}$ En esos años, Mosse mantuvo una actividad internacional frenética impartiendo constantemente conferencias y cursos en diversas universidades de prestigio por el mundo. Ciertamente, la obra era una fascinante lluvia de ideas e interpretaciones histó-

33. ("die Billigung des brutalen Tons der Nachkriegspolitik oder später für die Hinnahme der Nazi-Politik"), MOSSE, George L., "Der Erste Weltkrieg und die Brutalisierung der Politik. Betrachtungen über die politische Rechte, den Rassismus und den deutschen Sonderweg", en Manfred FUNKE, Hans-Adolf JACOBSEN, Hans-Helmuth KNÜTTER, Hans-Peter SCHWARZ (Hrgs.): Demokratie und Diktatur. Geist und Gestalt politischer Herrschaft in Deutschland und Europa, Bonn, Schriftenreihe der Bundeszentrale für politische Bildung, 1987, pp. 127-139, cita en p. 128.

34. Reseña de Fallen Soldiers por Robert Weldon Whalen en The Journal of Modern History, vol. 64, n 2 (1992), pp. 381-382.

35. Le guerre mondiali. Dalla tragedia al mito dei caduti, Roma-Bari, Laterza, 1990.

36. Gefallen für das Vaterland: nationales Heldentum und namenloses Sterben, Stuttgart, Klett/Kota, 1993.

37. Edición en ebreo: Tel Aviv, Am Oved, 1994; después fue publicado en japonés, con título Eirei: tsukurareta sekai taisen no kioku, Tokio, Kashiwa Shobo, 2001.

38. Otras reseñas positivas, por Jay W. Baird en American Historical Review, vol. 46, no 4 (1991), pp. 1160-1161; David R. Applebaum en The Journal of Military History, vol. 55, $n^{\circ} 1$ (1991), pp. 119-120; Arden Buchold en Central European History, vol. 24, no 2-3 (1991), pp. 187-194; Dennis E. Showalter en German Studies Review, vol. 13, nº 3 (1990), pp. 555-556; Peter Buitenhuis en Canadian Historical Review, vol. 72, no 3 (1991), p. 432; Annette Becker en Guerres mondiales et conflits contemporains, $\mathrm{n}^{\circ} 167$ (1992), p. 167; véase también la colección de recortes de prensa en Leo Baeck Institute (Nueva York), George L. Mosse Collection [LBIGMC], Series II: Writings and Research; Subseries 2: Reviews and reactions, Books, Fallen Soldiers, y Fallen Soldiers-German Version.

39. George L. Mosse a su editor en Jerusalén, 14 de enero de 1993, en LBIGMC, Subseries 2: Reviews and reactions, Series II: Writings and Research, Subseries 1: Writings, A) Books, d) Fallen Soldiers, Foreword to Israeli edition. 
ricas en torno a los mitos y representaciones de la Primera Guerra Mundial, y la tesis de la brutalización era simplemente una de ellas. De hecho, en un interesante intercambio epistolar mantenido entre Eric J. Leed y George L. Mosse, en el cual aquel historiador hacía crítica de diversos aspectos del libro, no hubo ninguna discusión de ese tema particular. ${ }^{40}$

\section{El debate sobre la brutalización}

Entre una general aclamación del libro Fallen Soldiers, la reacción muy crítica del historiador francés Antoine Prost, conocido por ser un referente clave de la historiografía contemporaneísta francesa y por su monumental obra publicada en 1977 sobre el movimiento excombatiente francés en el periodo de entreguerras (la cual Mosse conocía), ${ }^{41}$ marcó el comienzo del debate sobre la "brutalización". En 1994, con un artículo publicado en The Historical Journal, ${ }^{42}$ Prost puso en duda la proposición mosseana de la estrecha relación entre la experiencia de la Primera Guerra Mundial y el ascenso del nazismo, poniendo como contraejemplo el caso francés, donde según Prost el conjunto de la sociedad, y especialmente las organizaciones excombatientes de derecha e izquierda, fueron pacifistas durante el periodo de entreguerras. "Como los soldados franceses y alemanes lucharon la misma guerra", estas diferencias entre el pacifismo de unos y el belicismo de otros no podían deberse a "la guerra en sí", con su experiencia de muerte de masas, sino a procesos particulares en el seno de ambas sociedades. En Francia y Alemania existían, ya antes de la guerra, diferentes concepciones de la relación entre los individuos y la nación, distintos nacionalismos; por tanto la "brutalización" -esa "elevada indiferencia por la vida humana"- no podía ser la mera continuación de mentalidades bélicas en la paz, según Prost, sino la continuación de actitudes anteriores a 1914 en las condiciones de la posguerra. ${ }^{43}$ En ambos ejércitos se había

40. Leed a Mosse, 11 de septiembre de 1990, y Mosse a Leed, 20 de septiembre de 1990, en LBIGMC, Series II: Writings and Research; Subseries 2: Reviews and reactions, Books, Fallen Soldiers - Reactions.

41. PROST, Antoine, Les Anciens Combattants et la Societé Française 1914-1939, Paris, Presses de la Fondation Nationale des Sciences Politiques, 1977, 3 vols.; véase también Id., In the Wake of War. The 'Anciens Combattants' and French Society, Providence and Oxford, Berg, 1992.

42. PROST, Antoine, "The impact of war on French and German political cultures", The Historical Journal, vol. 37, nº 1 (1994), pp. 209-217.

43. James M. Diehl, en su estudio del paramilitarismo derechista en la Alemania de entreguerras había sugerido, ya en 1977, que estos grupos violentos y de excombatientes hundían sus raíces en la era bismarckiana: véase DIEHL, James M., Paramilitary politics in Weimar Germany, Bloomington, Indiana University Press, 1977. 
idealizado la figura del soldado como ideal de masculinidad, pero aquello sólo había conducido a la "brutalización" en Alemania y no en Francia. Además, entre los franceses, la experiencia de guerra en sí, según Prost, había sido la principal razón para rechazar la violencia; la acción de matar al enemigo nunca se habría legitimado tanto como en Alemania: la ética surgida de la guerra en Francia fue pacifista, de ahí que pudiera ponerse en duda que la "brutalización de la política alemana y su clímax nazi derivasen de la experiencia bélica": "La guerra no fue la principal causa del nacional-socialismo [escribió Prost]. La Primera Guerra Mundial fue suficientemente horrible como para cargarla con consecuencias innecesarias". ${ }^{4}$

A estas observaciones no hubo -que yo conozca- una respuesta directa por parte de Mosse, que entre tanto se había centrado en el estudio de la masculinidad y el nacionalismo judío y a una edad avanzada empezaba a considerar escribir sus memorias. Pero también otros historiadores durante la década de 1990 cuestionaron la validez de esa conexión entre experiencia de guerra y "brutalización". En 1993, Richard Bessel, que ya había investigado con detalle la violencia política nazi y consideraba "demasiado simples" las interpretaciones que la atribuían al efecto brutalizador de la guerra, publicó su pormenorizado estudio sobre la desmovilización de Alemania tras la Primera Guerra Mundial, en el que señalaba, por ejemplo, que muy pocos excombatientes habían sido aquellos inadaptados que se unieron al Freikorps..$^{45}$ En la misma línea, realizando un análisis muy documentado de la región de Baviera, Benjamin Ziemann argumentaría que la gran mayoría de veteranos de guerra estuvieron interesados en retornar a la pacífica normalidad de su vida rural, y no en continuar actuando en grupos paramilitares ${ }^{46}$ Estas perspectivas, además, se separaban de la corriente historiográfica culturalista sobre la Primera Guerra Mundial que adquirió fuerza esos años, y que solía asumir que el conflicto bélico había sido una cesura histórica clave, origen de la modernidad y de los totalitarismos. ${ }^{47}$

El debate sobre la "brutalización" se desenvolvió en un panorama historiográfico cada vez más complejo y amplio, dada la existencia de diferentes

44. PROST, Antoine, "The impact of war...", p. 217.

45. BESSEL, Richard, Germany after the First World War, Oxford, Oxford University Press, 1995, cita en p. vi.

46. ZIEMANN, Benjamin, Front und Heimat. Ländliche Kriegserfahrungen im südlichen Bayern 1914-1923, Essen, Klartext, 1997; traducido al inglés como Id., War Experiences in Rural Germany: 1914-1923, Oxford, Berg, 2007.

47. En Italia, la obra de GIBELLI, Antonio, L'officina della guerra. La Grande Guerra e le trasformazioni del mondo mentale, Torino, Bollati Bolinghieri, 1991, también se alineó con la perspectiva culturalista inaugurada por Fussell, Leed y Mosse. 
campos en los que el concepto -y la obra de Mosse- resultaba relevante: la memoria de la Primera Guerra Mundial, el paramilitarismo o violencia política posbélica, los excombatientes, el genocidio, o la controvertida "cultura de guerra". Además, el debate se internacionalizó sustancialmente: tras empezar siendo significativo en Alemania y entre germanistas, pasó a tener impacto entre los historiadores franceses. Numerosos especialistas italianos también recurrieron al concepto de "brutalización", que incluso terminó en manos de investigadores españoles para aplicarlo al estudio de un país que no participó en la Gran Guerra (España). En los últimos años, analistas de muy diversas procedencias, examinando la historia de países del este europeo y de otros continentes, han entrado en la misma discusión, prácticamente globalizada. Todo ello ocurrió desde mediados de la década de 1990, con gran intensidad durante el primer decenio del siglo XXI, y a lo largo del último lustro, pero es interesante destacar que la polémica sobre la "brutalización" tomó ímpetu justo después de que su catalizador, George L. Mosse, falleciera en 1999, coincidiendo también con la publicación de la traducción francesa de Fallen Soldiers (1999), y la reedición de la italiana (1998).

Sin duda, la vivacidad del debate intelectual en torno al impacto cultural de la Gran Guerra se debe en gran parte a la profusa actividad de los historiadores vinculados al Historial de Péronne, museo y centro de investigación fundado en 1989 (con participación de Mosse, Jay Winter, Gerd Krumeich y muchos otros) ${ }^{48}$ Varias obras de Stéphane Audoin-Rouzeau y Annette Becker son probablemente las más representativas de este grupo relativamente diverso. En su estudio del rol de la infancia en la "cultura de guerra" francesa, Audoin-Rouzeau ya aplicó el concepto de "brutalización", ${ }^{49}$ y en su prefacio a la edición francesa de Fallen Soldiers, este autor subrayó el interés y originalidad de esa tesis mosseana, que a pesar de ser "simple", nunca antes había sido propuesta por un historiador: el desconocimiento que Mosse claramente padecía respecto al caso francés -que podía contradecir su tesis- no era óbice para abrazar la general validez de la noción. Significativamente, la traducción francesa del libro incorporaba la palabra brutalisation al subtítulo, haciendo parecer más mecánica la interpretación mosseana de la evolución desde la

48. Véanse BECKER, Jean-Jacques, Jay M. WINTER, Gerd KRUMEICH, Annette BECKER, Stéphane AUDOIN-ROUZEAU (eds.), Guerre et Cultures 1914-1918, Paris, ArmandColin, 1994; WINTER, Jay, Sites of Memory, Sites of Mourning. The Great War in European cultural history, Cambridge, Cambridge University Press, 1995 (que subraya las continuidades, más que las novedades culturales traídas por la Gran Guerra); BECKER, Jean-Jacques (dir.), Histoire culturelle de la Grande Guerre, Paris, Armand Colin, 2005.

49. AUDOIN-ROUZEAU, Stéphane, La Guerre des enfants 1914-1918. Essai d'histoire culturelle, Paris, Colin, 1993, p. 11. 
"Gran Guerra al totalitarismo". ${ }^{50}$ Finalmente, Audoin-Rouzeau y Becker, en su importante obra 14-18, retrouver la Guerre, argumentaron, con Mosse, que la brutalización de las sociedades europeas en la Primera Guerra Mundial supuso efectivamente un retroceso del proceso civilizador, que explicaba el ascenso de los totalitarismos y la violencia transmitida por excombatientes hacia el mundo civil, no sólo en el nazismo, sino también en el comunismo soviético. ${ }^{51}$ Así, a la altura del año 2000, una reseñista de la edición francesa de Fallen Soldiers podía preguntarse retóricamente: “¿Cómo estudiar a día de hoy la Primera Guerra Mundial y el periodo de entreguerras sin recurrir al concepto de brutalization inventado por G. L. Mosse?". ${ }^{2}$

Pero la verdad es que hubo en Francia una fuerte reacción historiográfica contra la corriente de la culture de guerre, aunque haya sido menos prominente. ${ }^{53}$ Diversos historiadores (Rémy Cazals, Nicolas Mariot, Nicolas Offenstadt, etc.) han puesto de relieve el disenso de muchos soldados frente a las prácticas de violencia de guerra, y el pacifismo de algunos combatientes y veteranos. Por su parte, Antoine Prost se encargó de subrayar los "límites de la brutalización", en un artículo publicado en la prestigiosa revista Vingtième Siècle: la experiencia de matar habría sido muy poco común entre los soldados del frente occidental, y la experiencia de guerra no habría cambiado mucho la personalidad de aquellos que la combatieron. ${ }^{54}$ Con todo, este artículo de Prost, menos confrontacional que su crítica de 1994, destaca por asumir plenamente la validez del concepto de "brutalización", aceptado ahora como un fenómeno histórico que realmente sucedió, por mucho que Prost pusiera límites su alcance sobre todo a raíz del caso francés. Esta consolidación de la noción de "brutalización" en el lenguaje historiográfico como un concepto al que se le atribuye capacidad analítica y explicativa, a pesar de críticas y matices, es -en mi opinión- el resultado más importante del debate.

50. MOSSE, George L. De la Grande Guerre au totalitarisme. La brutalisation des sociétés européennes, Paris, Hachette, 1999, pp. xiv-xv.

51. AUDOIN-ROUZEAU, Stéphane y Annette BECKER, 14-18, retrouver la Guerre, Paris, Gallimard, 2000, pp. 58-60, 227-228, 313-314.

52. ("Comment étudier aujourd'hui la première guerre mondiale et l'entre-deux-guerres sans avoir recours au concept de brutalization inventé par G.L. Mosse ?") Reseña de Raphaëlle Branche en Vingtième Siècle. Revue d'histoire, num. 66 (2000), p. 185.

53. Sobre el debate véase SMITH, Leonard V., "The 'Culture de guerre' and French Historiography of the Great War of 1914-1918", History Compass, Vol. 5, no 6 (2007); PURSEIGLE, Pierre, "A very French debate: the 1914-1918 'war culture", Journal of War and Culture Studies, vol. 1, no 1 (2008), pp. 9-14; Frédéric Rousseau, "Repensar la gran guerra (1914-1918). Historia, testimonios y ciencias sociales", Historia Social, no 78 (2014), pp. 135-153.

54. PROST, Antoine, "Les limites de la brutalisation. Tuer sur le front occidental, 1914-1918", Vingtième Siècle. Revue d'histoire, $\mathrm{n}^{\circ} 81$ (2004), pp. 5-20. 
La verdad es que en los años 2000 hubo un auténtico torrente de libros que abrazaban, más o menos explícitamente, la tesis de la "brutalización", y exploraban ese continuum cultural entre una guerra y la otra: no sólo en la historiografía francesa ${ }^{55}$, sino también en el ámbito anglófono ${ }^{56}$ y entre los historiadores italianos y alemanes. ${ }^{57}$ Un volumen colectivo publicado en París en 2004 directamente afirmaba en su introducción querer abordar esa cuestión. ${ }^{58}$ En España, hubo quien empezó a aplicar la noción de "brutalización" para describir la violencia política de la etapa de la Segunda República. ${ }^{59}$ El concepto de "brutalización" incluso pasó a estudiarse en algunas escuelas secundarias francesas, como un proceso histórico que así se ha equiparado a otras nociones clave en las programaciones educativas (como globalización, desarrollo o totalitarismo). ${ }^{60}$

Pero al costado de todas esas obras de óptica culturalista, también durante los 2000 se realizaron importantes estudios centrados en la violencia política y el paralimilitarismo de entreguerras, que demostraron una realidad mucho más rugosa y confusa que la transmitida por la idea de "brutalización". La his-

55. AUDOIN-ROUZEAU, Stéphane, Annette BECKER, Christian INGRAO, Henry ROUSSO, La violence de guerre 1914-1945. Approches comparées des deux conflits mondiaux, Paris, IHTP-CNRS, 2002; CABANES, Bruno y Édouard HUSSON (coords.), Les sociétés en guerre 1911-1946, Paris, Armand Colin, 2003.

56. BARTOV, Omer, Mirrors of Destruction. War, Genocide, and Modern Identity, Oxford et al., Oxford University Press, 2000, pp. 16-22; KRAMER, Alan, Dynamic of Destruction. Culture and Mass Killing in the First World War, Oxford and New York, Oxford University Press, 2007.

57. VENTRONE, Angelo, La seduzione totalitaria. Guerra, modernita, violenza politica (1914-1918), Roma, Donzelli, 2003; TRAVERSO, Enzo, A ferro e fuoco. La guerra civile europea, 1914-1945, Bologna, Il Mulino, 2007; MESCHING, Alexander, Der Wille zur Bewegung. Militarischer Traum und totalitares Programm. Eine Mentalitatsgeschichte vom Ersten Weltkrieg zum Nationalsozialismus, Bielefeld, Transcript, 2008. Algo anterior, el estudio comparativo de la violencia política en París y Berlín durante el periodo de entreguerras de WIRSCHING, Andreas, Vom Weltkrieg zum Bürgerkrieg? Politischer Extremismus in Deutschland und Frankreich, 1918-1933/39. Berlin und Paris im Vergleich, Munich, R. Oldenbourg, 1999, también se apoyaba en Mosse (p. 5).

58. CAUSARANO, Pietro, Valeria GALIMI, François GUEDJ, Romain HURET, Isabelle LESPINET-MORET, Jérôme MARTIN, Michel PINAULT, Xavier VIGNA, Mercedes YUSTA, Le XXe siècle des guerres, Paris, Les Éditions de l'Atelier/Éditions Ouvrières, 2004, p. 14 y passim.

59. REY REGUILLO, Fernando del y Mercedes CABRERA, "La patronal y la brutalización de la política", en Santos JULIÁ (coord.), Violencia política en la España del siglo XX, Madrid, Taurus, 2000, p. 235-288; DEL REY, Fernando (dir.), Palabras como puños. La intransigencia política en la Segunda República española, Madrid, Tecnos, 2011. Sobre la tardía recepción de la obra de Mosse en España véase ALCALDE, Ángel, "Soldados caídos. Un estudio introductorio", en MOSSE, George L., Soldados Caídos, op. cit., pp. 9-27.

60. http://histoire-geo.ac-amiens.fr/?Brutalisation\&lang=fr [consultado: 13-X-2015] . 
toriografía alemana (mucho más dada a la exhaustividad en el trabajo de fuentes de archivo que, por ejemplo, la norteamericana) produjo resultados más complejos. Así, un número especial del Journal of Modern European History, introducido por Dirk Schumann -especialista en la violencia política durante la República de Weimar-, tras explorar la "continuidad" de la violencia en la posguerra europea, concluyó que no había sido la experiencia de guerra en sí, sino las culturas políticas que la interpretaron, lo que había provocado la violencia política. ${ }^{61}$ Además, el mapa de la violencia en el continente revelaba un patrón por el cual los estados-nación más jóvenes, particularmente en el este de Europa, sufrieron el paramilitarismo mucho más intensamente, al derrumbarse la autoridad del Estado. Ni siquiera en Alemania, según Schumann, la lucha guerracivilista de 1918-1921 podía atribuirse tampoco a una mera "brutalización" producida por la Primera Guerra Mundial. ${ }^{62}$

Aún así, el concepto de "brutalización" marcó en el último decenio buena parte de la agenda investigadora de los especialistas en la violencia política del periodo de entreguerras europeo. Respecto al caso italiano, por ejemplo, aunque a veces no hubiera una innovación metodológica sustancial desde lo ya aportado por Emilio Gentile en su estudio sobre el movimiento fascista, ${ }^{63}$ se incorporó la noción de brutalizzazione para explicar su ascenso en el periodo 1919-1922. ${ }^{64} \mathrm{Y}$ solapándose con este campo, los estudios sobre los procesos de desmovilización bélica también han recurrido al concepto mosseano.$^{65} \mathrm{En}$ su investigación sobre el paramilitarismo contrarrevolucionario en Europa central, Robert Gerwarth confirmó que "exoficiales brutalizados por la guerra y furiosos por la derrota y la revolución se unieron a miembros de una generación más joven" sin experiencia de guerra, para ejercer una violencia extrema de

61. SCHUMANN, Dirk, "Europa, der Erste Weltkrieg und die Nachkriegszeit: eine Kontinuität der Gewalt?", Journal of Modern European History, Vol. 1, Num. 1 (2003), pp. 24-43. El dossier contiene artículos de Adrian Gregory, Benjamin Ziemann, Andreas Wirsching, y Piotr Wróbel.

62. SCHUMANN, Dirk, Politische Gewalt in der Weimarer Republik: Kampf um die Straße und Furcht vor dem Bürgerkrieg, Essen, Klartext, 2001. En su traducción inglesa Id., Political Violence in the Weimar Republic: Fight for the Streets and Fear of Civil War, New York, Berghahn Books, 2009, especialmente pp. viii, xvi, 306.

63. GENTILE, Emilio, Storia del partito fascista. 1919-1922. Movimento e milizia, RomaBari,Laterza, 1989.

64. ARAMINI, Donatello, George L. Mosse..., pp. 229-232; ALBANESE, Giulia, "La brutalizzazione della politica tra guerra e dopoguerra", Contemporanea, a. IX, num. 3 (2006), pp. 551-557; MONDINI, Marco, La politica delle armi. Il ruolo dell'esercito nell'avvento del fascismo, Roma-Bari, Laterza, 2006, p. 54; ALBANESE, Giulia, La marcia su Roma, Roma-Bari, Laterza, 2006, pp. 204-206.

65. MONDINI, Marco y Guri SCHWARZ, Dalla guerra alla pace. Retoriche e pratiche della smobilitazione nell'Italia del Novecento, Verona, Cierre edizioni / Istrevi, 2007, p. 12. 
carácter anticomunista, misógino y antisemita entre 1918 y 1920, y que continuó ocasionalmente más allá de estas fechas. ${ }^{66}$

Desde las perspectivas sobre los procesos de desmovilización bélica provinieron nuevos conceptos que han permitido a muchos historiadores refinar la tesis mosseana de la "brutalización". Ya en 2001, John Horne propuso la noción de "desmovilización cultural" para poder entender porqué en unos países, normalmente victoriosos, las mentalidades bélicas se desarticularon, mientras que en otros lugares, sobre todo Alemania, persistieron. ${ }^{67}$ En Francia, por ejemplo, los poilus habrían abandonado lentamente las representaciones mentales propias de la agresiva "cultura de guerra". ${ }^{68}$ Lo que en francés se ha llamado "sortie de guerre" habría resultado teóricamente un proceso crucial, ${ }^{69}$ que acabaría explicando por qué en unos países hubo "brutalización" y en otros no. No obstante, estudios detallados y de fuerte base archivística todavía encuentran dificultades para evidenciar los procesos, fallidos o exitosos, de desmovilización cultural, y extraer conclusiones de ellos. ${ }^{70}$

\section{Las últimas contribuciones al debate}

En el último lustro, tras muchas controversias con ecos cada vez más internacionales, y con un concepto de "brutalización" muy enraizado en el discurso historiográfico, se han propuesto algunos usos muy matizados de la famosa tesis mosseana. Por ejemplo Arnd Weinrich ha propuesto la existencia de una "brutalización primaria" en las trincheras, y una "secundaria" y "terciaria" que se habría producido en la educación militarista dada en la sociedad posbélica y particularmente en las Juventudes Hitlerianas. ${ }^{71}$ También debemos destacar el trabajo colectivo de los historiadores vinculados al Centre for War Studies

66. GERWARTH, Robert, "The Central European Counter-Revolution: Paramilitary Violence in Germany, Austria and Hungary after the Great War", Past and Present, ${ }^{\circ}$ 200 (2008), pp. 175-209.

67. HORNE, John (dir.), "Demobilisations culturelles apres la Grande Guerre", 14-18 Aujourd'hui, Today, Heute, no 5 (2002).

68. CABANES, Bruno, La Victoire endeuillée. La sortie de guerre des soldats français (19181920), Seuil, Paris, 2004.

69. CLAISSE, Stéphanie y Thierry LEMOINE (coords.), Comment (se) sortir de la Grande Guerre? Regards sur quelques pays "vainqueurs »: la Belgique, la France et la GrandeBretagne, Paris, LHarmattan, 2005; AUDOIN-ROUZEAU, Stéphane y Christoph PROCHASSON (dirs.), Sortir de la Grande Guerre. Le monde et l'apres 1918, Paris, Tallandier, 2008.

70. SEIPP, Adam R., The Ordeal of Peace. Demobilization and the Urban Experience in Britain and Germany, 1917-1921, Farnham, Ashgate, 2009, p. 22.

71. WEINRICH, Arndt, Der Weltkrieg als Erzieher. Jugend zwischen Weimarer Republik und Nationalsozialismus, Essen, Klartext Verlag, 2013, pp. 21-25. 
(University College Dublin), en el marco del proyecto "Los límites de la desmovilización. Violencia paramilitar en Europa y el mundo, 1915-1923". Su investigador principal, Robert Gerwarth, ha afirmado que la "brutalización" no se debió a la experiencia de guerra en sí, sino a la derrota y la subsiguiente revolución que tuvo lugar en diversas partes del centro y este del continente. ${ }^{72}$ Siguiendo esta línea, se ha añadido que la violencia ejercida muy a menudo por excombatientes fue posible en un marco de crisis de la autoridad del estado. ${ }^{73}$ Como afirma un artículo que revisita la tesis de la "brutalización" en el caso de Rusia, "es difícil establecer conexiones directas entre los frentes de una guerra industrializada y la violencia de la guerra civil y la revolución". ${ }^{74}$

Últimamente, junto a la noción de "desmovilización cultural" se ha recurrido al concepto de "cultura de la derrota" (propuesto originalmente por Wolfgang Schivelbusch, ${ }^{75}$ y recogido por John Horne, Robert Gerwarth y otros), para explicar que unos países sufriesen la "brutalización" de la política y otros no tras 1918, a pesar de que sea difícil documentar dicho fenómeno. Según Julia Eichenberg y John Paul Newman en su obra sobre el internacionalismo y pacifismo de grupos excombatientes durante el periodo de entreguerras, la evolución de las "culturas de la victoria" por un lado, y las "culturas de la derrota", por otro, explica que en países como Francia e Inglaterra se desarrollara entre los veteranos una cultura de paz, y en Alemania y los países derrotados una cultura de guerra, haciendo encajar en ello el caso italiano, que sin embargo requiere mucho mayor estudio. ${ }^{76}$

72. GERWARTH, Robert, Fighting the Red Beast: Counter-Revolutionary Violence in the Defeated States of Central Europe, en Robert GERWARTH y John HORNE (eds.), War in Peace. Paramilitary Violence in Europe after the Great War, Oxford, Oxford University Press, 2012, pp. 52-71 (el volumen contiene también aportaciones de Tomas Balkelis, Anne Dolan, Julia Eichenberg, Emilio Gentile, Pertty Haapala, John Horne, John Paul Newman, William Rosenberg, Marko Tikka, Ugur Ümit Üngör, Serhy Yekelchyk); véase también GERWARTH, Robert y John HORNE, "Vectors of Violence: Paramilitarism in Europe after the Great War, 1917-1923", The Journal of Modern History, vol. 83, no 3 (2011), pp. 489-512.

73. EDELE, Mark y Robert GERWARTH (eds.), dossier "The Limits of Demobilization", Journal of Contemporary History, vol. 50, $\mathrm{n}^{\circ} 1$ (2015), con artículos de Dietrich BEYRAU, Tomas Balkelis, Jochen Böhler, Jennifer Keene, Richard S. Fogarty y David Killingray, y Stephen Garton; véase también EICHENBERG, Julia, "The Dark Side of Independence: Paramilitary Violence in Ireland and Poland after the First World War", Contemporary European History, vol. 19, n 3 (2010), pp. 231-248.

74. BEYRAU, Dietrich, "Brutalization revisited: the case of Russia", Journal of Contemporary History, vol. 50, $\mathrm{n}^{\circ} 1$ (2015), pp. 15-37, cita en p. 16.

75. SCHIVELBUSCH, Wolfgang, Die Kultur der Niederlage. Die amerikanische Suden 1986. Frankreich 1871. Deutschland 1918, Berlin, Alexander Fest, 2001.

76. EICHENBERG, Julia y John Paul NEWMAN (eds.), The Great War and Veterans' Internationalism, New York, Palgrave, 2013. 
En realidad, como viene siendo cada vez más notorio, las perspectivas sobre el periodo de entreguerras europeo que sencillamente yuxtaponen una serie de casos nacionales estancos, atribuyendo a unos la etiqueta de "brutalizados" y a otros la de "pacifistas", sólo conducen a contradicciones y a nuevos interrogantes, sobre todo si se introducen casos sustancialmente ignorados, como el español, ${ }^{77}$ o se profundizan y reexaminan otros. ${ }^{78}$ De hecho, una reciente revisión de la historia del movimiento excombatiente francés ha puesto en duda la interpretación dominante sobre su pacifismo mayoritario y su efectiva "desmovilización cultural", ${ }^{79}$ y Benjamin Ziemann, al valorizar las actividades de los excombatientes alemanes pro-republicanos, ha seguido insistiendo en su rechazo de la tesis de la "misteriosa brutalización bélica" en la Alemania de Weimar. ${ }^{80}$

En este panorama en el que la tesis de la "brutalización" parece haber agotado su potencial, se han explorado nuevas maneras de entender los vínculos entre la experiencia de guerra, la violencia política de posguerra, los excombatientes, el paramilitarismo y los fascismos. Yo he propuesto la aplicación de conceptos provenientes de la ciencia política, en particular los procesos de DDR (desmovilización, desarme, reintegración), como microscopio con el que reexaminar el periodo de entreguerras, y he sugerido que los altos niveles de sangrienta violencia política en lugares como Italia o Alemania en el periodo 19191923 se debieron, entre otros factores, a una inusitada disponibilidad de armas de fuego, causada por un fracaso en el proceso posbélico de "desarme". ${ }^{81}$ Sobre todo, he argumentado que la relación entre los excombatientes y el fascismo en el periodo de entreguerras europeo no puede comprenderse a partir de la tesis de la "brutalización", la cual además de ser inverificable deja muchas

77. Cabe lamentar el general desconocimiento internacional de los múltiples trabajos sobre el paramilitarismo y la violencia política en España por parte Eduardo González Calleja, que sugirió el interés de aplicar el concepto de "brutalización" al caso español: GONZÁLEZ CALLEJA, Eduardo, "La cultura de guerra como propuesta historiográfica; una reflexión general desde el contemporaneísmo español", Historia Social, n 61 (2008), pp. 69-87.

78. ALCALDE FERNÁNDEZ, Ángel, "Experiencias de guerra y fascismos: los excombatientes en Europa y España (1914-1945). Una introducción comparativa", en Carmen FRÍAS, José Luis LEDESMA, Javier RODRIGO (eds.): Reevaluaciones. Historias locales, miradas globales (VII Congreso de Historia Local de Aragón), Zaragoza, Institución "Fernando el Católico", 2011, pp. 365-376.

79. MILLINGTON, Chris, From Victory to Vichy. Veterans in Inter-war France, Manchester and New York, Manchester University Press, 2012.

80. ZIEMANN, Benjamin, Contested Commemorations. Republican War Veterans and Weimar Political Culture, Cambridge et alii, Cambridge University Press, 2013, p. 11.

81. ALCALDE, Ángel, "Excombatientes, ¿germen de guerras civiles?", Amnis (2015) https:// amnis.revues.org/2452 [consultado: 13-X-2015]. 
dimensiones entre tinieblas. Desde una perspectiva transnacional, mi tesis doctoral demuestra que la relación entre los veteranos de guerra y los movimientos y regímenes fascistas se basó en un proceso de apropiación simbólica del propio concepto de "excombatiente", del que el fascismo italiano fue su máximo beneficiario, dando lugar a interconectados procesos de transnacionalización del fascismo y fascistización de la política excombatiente en Europa ${ }^{82}$ La noción de "brutalización", bajo mi punto de vista, alberga solamente un potencial descriptivo y no analítico.

\section{Conclusión}

Con todo, parece claro que todavía la noción mosseana de "brutalización" seguirá siendo profusamente utilizada en diversas historiografías, aunque solamente sea como manera de adjetivar la persistente violencia política del periodo de entreguerras europeo. El fenómeno histórico de la "brutalización", si por un lado se manifiesta como una verdad aparentemente evidente vista desde lejos, ha resultado imposible o extremadamente difícil de demostrar de manera sistemática a partir de fuentes documentales. Este artículo ha demostrado que el recurso a explicar los elevados niveles de violencia y conflictividad posbélica a través del efecto "brutalizador" atribuido a la experiencia de guerra no fue una invención de George L. Mosse, ni siquiera por la utilización de ese falso neologismo: la tesis de la "brutalización" ya existía, a su manera, antes de la Primera Guerra Mundial, y no es difícil encontrarla expresada incluso con esa palabra en fuentes documentales tras 1918, para referirse a las consecuencias negativas de la participación en la guerra, sobre todo entre los excombatientes. Mosse la rescató, considerándola una manera convincente y muy sugerente de explicar el por qué del genocidio y del ascenso del nazismo en Alemania. La "brutalización", en efecto, es un atajo, muy retórico y figurativo, para transmitir cómo se pudo repetir, multiplicada, la tragedia de 19141918. Comparaciones, análisis pormenorizados y otras metodologías a menudo han puesto en duda mucho de lo que Mosse afirmó, pero el más importante resultado del debate ha sido la consolidación del uso del concepto, junto a propuestas más matizadas de su tesis. No obstante, más de veinticinco años después de la publicación de Fallen Soldiers, tras poner de relieve la gran contribución de este clásico a nuestra manera de pensar la Primera Guerra Mundial y sus consecuencias, los historiadores del periodo de entreguerras deberían

82. ALCALDE, Ángel, War Veterans and Transnational Fascism. From Fascist Italy and Nazi Germany to Francoist Spain and Vichy France (1917-1940), PhD dissertation, European University Institute, 2015. 
recurrir a otras herramientas heurísticas para explicar los procesos por los que se llegó, realmente, al ascenso de los fascismos en diferentes países europeos, al genocidio y a la Segunda Guerra Mundial.

\section{BIBLIOGRAFÍA}

ALBANESE, Giulia, La marcia su Roma, Roma-Bari, Laterza, 2006.

ALBANESE, Giulia, "La brutalizzazione della politica tra guerra e dopoguerra", Contemporanea, a. IX, no 3 (2006).

ALCALDE FERNÁNDEZ, Ángel, "Experiencias de guerra y fascismos: los excombatientes en Europa y España (1914-1945). Una introducción comparativa", en Carmen FRÍAS, José Luis LEDESMA, Javier RODRIGO (eds.): Reevaluaciones. Historias locales, miradas globales (VII Congreso de Historia Local de Aragón), Zaragoza, Institución "Fernando el Católico", 2011, pp. 365-376.

ALCALDE, Ángel, "Excombatientes, ¿germen de guerras civiles?", Amnis (2015).

ALCALDE, Ángel, War Veterans and Transnational Fascism. From Fascist Italy and Nazi Germany to Francoist Spain and Vichy France (1917-1940), PhD dissertation, European University Institute, 2015.

ARAMINI, Donatello, George L. Mosse, LItalia e gli storici, Franco Angeli, Milano, 2010.

ASCHHEIM, Steven E., "George Mosse at 80: A Critical Laudatio", Journal of Contemporary History, vol. 34, no 2 (1999), pp. 295-312.

AUDOIN-ROUZEAU, Stéphane, La Guerre des enfants 1914-1918. Essai d'histoire culturelle, Paris, Colin, 1993.

AUDOIN-ROUZEAU, Stéphane y Annette BECKER, 14-18, retrouver la Guerre, Paris, Gallimard, 2000.

AUDOIN-ROUZEAU, Stéphane, Annette BECKER, Christian INGRAO, Henry ROUSSO, La violence de guerre 1914-1945. Approches comparées des deux conflits mondiaux, Paris, IHTP-CNRS, 2002.

AUDOIN-ROUZEAU, Stéphane y Christoph PROCHASSON (dirs.), Sortir de la Grande Guerre. Le monde et l'apres 1918, Paris, Tallandier, 2008.

BAILEY, William C., "Deterrence, Brutalization, and the Death Penalty: Another Examination of Oklahoma's Return to Capital Punishment", Criminology, vol. 36, $\mathrm{n}^{\mathrm{o}} 4$ (1998), pp. 711-734.

BARTOV, Omer, Mirrors of Destruction. War, Genocide, and Modern Identity, Oxford et al., Oxford University Press, 2000.

BECKER, Jean-Jacques, Jay M. WINTER, Gerd KRUMEICH, Annette BECKER, Stéphane AUDOIN-ROUZEAU (eds.), Guerre et Cultures 1914-1918, Paris, ArmandColin, 1994.

BECKER, Jean-Jacques (dir.), Histoire culturelle de la Grande Guerre, Paris, Armand Colin, 2005.

BESSEL, Richard, Germany after the First World War, Oxford, Oxford University Press, 1995. 
BEYRAU, Dietrich, "Brutalization revisited: the case of Russia", Journal of Contemporary History, vol. 50, nº 1 (2015), pp. 15-37

CABANES, Bruno y Édouard HUSSON (coords.), Les sociétés en guerre 1911-1946, Paris, Armand Colin, 2003.

CABANES, Bruno, La Victoire endeuillée. La sortie de guerre des soldats français (19181920), Seuil, Paris, 2004.

CAUSARANO, Pietro, Valeria GALIMI, François GUEDJ, Romain HURET, Isabelle LESPINET-MORET, Jérôme MARTIN, Michel PINAULT, Xavier VIGNA, Mercedes YUSTA, Le XXe siècle des guerres, Paris, Les Éditions de l'Atelier/Éditions Ouvrières, 2004.

CLAISSE, Stéphanie y Thierry LEMOINE (coords.), Comment (se) sortir de la Grande Guerre? Regards sur quelques pays « vainqueurs »: la Belgique, la France et la GrandeBretagne, Paris, LHarmattan, 2005.

DEL REY, Fernando (dir.), Palabras como puños. La intransigencia política en la Segunda República española, Madrid, Tecnos, 2011.

DIEHL, James M., Paramilitary politics in Weimar Germany, Bloomington, Indiana University Press, 1977.

EDELE, Mark y Robert GERWARTH (eds.), dossier "The Limits of Demobilization", Journal of Contemporary History, vol. 50, no 1 (2015).

EICHENBERG, Julia, "The Dark Side of Independence: Paramilitary Violence in Ireland and Poland after the First World War", Contemporary European History, vol. 19, no 3 (2010), pp. 231-248.

EICHENBERG, Julia y John Paul NEWMAN (eds.), The Great War and Veterans' Internationalism, New York, Palgrave, 2013.

EKSTEINS, Modris, Rites of Spring. The Great War and the Birth of the Modern Age, Boston, Houghton Mifflin Company, 1989.

EKSTEINS, Modris, La consagración de la primavera. La Gran Guerra y el nacimiento de los tiempos modernos, Valencia, Pre-Textos, 2014.

ELIAS, Norbert, "Civilización y violencia”, Reis, no 65 (1994), pp. 141-151

FUSSELL, Paul, The Great War and Modern Memory, Oxford, Oxford University Press, 1975

FUSSELL, Paul, La Gran guerra y la memoria moderna, Madrid, Turner, 2006.

GENTILE, Emilio, Storia del partito fascista. 1919-1922. Movimento e milizia, RomaBari, Laterza, 1989.

GENTILE, Emilio, Il fascino del persecutore. George L. Mosse e la catastrofe dell'uomo moderno, Roma, Carocci, 2007.

GERWARTH, Robert, "The Central European Counter-Revolution: Paramilitary Violence in Germany, Austria and Hungary after the Great War", Past and Present, ${ }^{\circ}$ 200 (2008), pp. 175-209.

GERWARTH, Robert y John HORNE, "Vectors of Violence: Paramilitarism in Europe after the Great War, 1917-1923", The Journal of Modern History, vol. 83, n 3 (2011), pp. 489-512. 
GERWARTH, Robert, Fighting the Red Beast: Counter-Revolutionary Violence in the Defeated States of Central Europe, en Robert GERWARTH y John HORNE (eds.), War in Peace. Paramilitary Violence in Europe after the Great War, Oxford, Oxford University Press, 2012, pp. 52-71.

GIBELLI, Antonio, L'officina della guerra. La Grande Guerra e le trasformazioni del mondo mentale, Torino, Bollati Bolinghieri, 1991.

GONZÁLEZ CALLEJA, Eduardo, "La cultura de guerra como propuesta historiográfica; una reflexión general desde el contemporaneísmo español”, Historia Social, Num. 61 (2008), pp. 69-87.

GRIFFIN, Roger, "Fascism and Culture: A Mosse-Centric Meta-Narrative (or how Fascist Studies Reinvented the Wheel)", en António COSTA-PINTO, ed., Rethinking the Nature of Fascism. Comparative Perspectives, Basingstoke, Palgrave Macmillan, 2011, pp. 85-116.

HORNE, John (dir.), "Demobilisations culturelles apres la Grande Guerre", 14-18 Aujourd'hui, Today, Heute, no 5 (2002).

JONES, Nigel H., Hitler's Heralds. The Story of the Freikorps, 1918-1923, London, Murray, 1987

KEEGAN, John, The Face of Battle, London, Jonathan Cape, 1976.

KEEGAN, John, El rostro de la batalla, Madrid, Ediciones Ejército, 1990.

KING, David R., "The Brutalization Effect: Execution Publicity and the Incidence of Homicide in South Carolina", Social Forces, vol. 57, no 2 (1978), pp. 683-687.

KRAMER, Alan, Dynamic of Destruction. Culture and Mass Killing in the First World War, Oxford and New York, Oxford University Press, 2007.

LAWRENCE, Jon, "Forging a Peaceable Kingdom: War, Violence, and Fear of Brutalization in Post-First World War Britain", The Journal of Modern History, vol. 75, n 3 (2003), pp. 557-589.

LEED, Eric J., No Man's Land. Combat and Identity in World War I, Cambridge, Cambridge University Press, 1979.

LEONI, Diego y Camillo ZADRA (eds.), La Grande Guerra: Esperienza, memoria, immagini, Bologna, Il Mulino, 1986.

MESCHING, Alexander, Der Wille zur Bewegung. Militarischer Traum und totalitares Programm. Eine Mentalitatsgeschichte vom Ersten Weltkrieg zum Nationalsozialismus, Bielefeld, Transcript, 2008.

MILLINGTON, Chris, From Victory to Vichy. Veterans in Inter-war France, Manchester and New York, Manchester University Press, 2012.

MONDINI, Marco, La politica delle armi. Il ruolo dell'esercito nell'avvento del fascismo, Roma-Bari, Laterza, 2006.

MONDINI, Marco y Guri SCHWARZ, Dalla guerra alla pace. Retoriche e pratiche della smobilitazione nell'Italia del Novecento, Verona, Cierre edizioni / Istrevi, 2007.

MOSSE, George L. y Michael A. LEDEEN, eds., Intervista sul nazismo, Roma-Bari, Laterza, 1977. 
MOSSE, George L., Nazism. A Historical and Comparative Analysis of National Socialism, Oxford, Basil Blackwell, 1978.

MOSSE, George L., "La sinistra europea e l'esperienza della guerra (Germania e Francia)", en Rivoluzione e Reazione in Europa 1917/1924. Convegno storico internazionale - Perugia 1978, Roma, Mondo Operaio - Edizioni Avanti!, 1978, vol. II, pp. 151-167

MOSSE, George L., "National Cemeteries and National Revival: The Cult of the Fallen Soldiers in Germany", Journal of Contemporary History, vol. 14, $\mathrm{n}^{\circ} 1$ (Jan. 1979), pp. 1-20.

MOSSE, George L. "Two World Wars and the Myth of the War Experience", Journal of Contemporary History, vol. 21, no 4 (1986), pp. 491-513.

MOSSE, George L., "Der Erste Weltkrieg und die Brutalisierung der Politik. Betrachtungen über die politische Rechte, den Rassismus und den deutschen Sonderweg", en Manfred FUNKE, Hans-Adolf JACOBSEN, Hans-Helmuth KNÜTTER, Hans-Peter SCHWARZ (Hrgs.): Demokratie und Diktatur. Geist und Gestalt politischer Herrschaft in Deutschland und Europa, Bonn, Schriftenreihe der Bundeszentrale für politische Bildung, 1987, pp. 127-139.

MOSSE, George L., Fallen Soldiers. Reshaping the Memory of the World Wars, New York, Oxford University Press, 1990.

MOSSE, George L. De la Grande Guerre au totalitarisme. La brutalisation des sociétés européennes, Paris, Hachette, 1999.

MOSSE, George L., Confronting History. A memoir, Madison, University of Wisconsin, 2000.

MOSSE, George L., Soldados caídos. La transformación de la memoria de las guerras mundiales, Zaragoza, Prensas de la Universidad de Zaragoza, 2016.

PIERACCINI, Paolo, Il Corpo di Polizia Municipale di Firenze. Dai Lorena all'Itaia repubblicana, Florencia, Paganini, 2004.

PLESSINI, Karel, The Perils of Normalcy. George L. Mosse and the Remaking of Cultural History, Madison, University of Wisconsin Press, 2014.

PROST, Antoine, Les Anciens Combattants et la Societé Française 1914-1939, Paris, Presses de la Fondation Nationale des Sciences Politiques, 1977

PROST, Antoine, "The impact of war on French and German political cultures", The Historical Journal, vol. 37, no 1 (1994), pp. 209-217.

PROST, Antoine, "Les limites de la brutalisation. Tuer sur le front occidental, 19141918", Vingtième Siècle. Revue d'histoire, no 81 (2004), pp. 5-20.

PURSEIGLE, Pierre, "A very French debate: the 1914-1918 'war culture", Journal of War and Culture Studies, vol. 1, no 1 (2008), pp. 9-14.

REY REGUILLO, Fernando del y Mercedes CABRERA, "La patronal y la brutalización de la política", en Santos JULIÁ (coord.), Violencia política en la España del siglo XX, Madrid, Taurus, 2000, p. 235-288.

ROUSSEAU, Frédéric, "Repensar la gran guerra (1914-1918). Historia, testimonios y ciencias sociales", Historia Social, nº 78 (2014), pp. 135-153. 
SCHIVELBUSCH, Wolfgang, Die Kultur der Niederlage. Die amerikanische Suden 1986. Frankreich 1871. Deutschland 1918, Berlin, Alexander Fest, 2001

SCHUMANN, Dirk, Politische Gewalt in der Weimarer Republik: Kampf um die Straße und Furcht vor dem Bürgerkrieg, Essen, Klartext, 2001.

SCHUMANN, Dirk, Political Violence in the Weimar Republic: Fight for the Streets and Fear of Civil War, New York, Berghahn Books, 2009

SCHUMANN, Dirk, "Europa, der Erste Weltkrieg und die Nachkriegszeit: eine Kontinuität der Gewalt?", Journal of Modern European History, Vol. 1, Num. 1 (2003), pp. 24-43.

SEIPP, Adam R., The Ordeal of Peace. Demobilization and the Urban Experience in Britain and Germany, 1917-1921, Farnham, Ashgate, 2009.

SMITH, Leonard V., "The 'Culture de guerre' and French Historiography of the Great War of 1914-1918”, History Compass, Vol. 5, n 6 (2007).

TRAVERSO, Enzo, A ferro e fuoco. La guerra civile europea, 1914-1945, Bologna, Il Mulino, 2007.

VENTRONE, Angelo, La seduzione totalitaria. Guerra, modernita, violenza politica (1914-1918), Roma, Donzelli, 2003.

WARD, Stephen R., ed., The War Generation. Veterans of the First World War, London y Port Washington (NY), Kennikat Press, 1975.

WEINRICH, Arndt, Der Weltkrieg als Erzieher. Jugend zwischen Weimarer Republik und Nationalsozialismus, Essen, Klartext Verlag, 2013.

WINTER, Jay, Sites of Memory, Sites of Mourning. The Great War in European cultural history, Cambridge, Cambridge University Press, 1995.

WIRSCHING, Andreas, Vom Weltkrieg zum Bürgerkrieg? Politischer Extremismus in Deutschland und Frankreich, 1918-1933/39. Berlin und Paris im Vergleich, Munich, R. Oldenbourg, 1999.

WOHL, Robert, The Generation of 1914, Cambridge, Ma., Harvard University Press, 1979.

ZIEMANN, Benjamin, Front und Heimat. Ländliche Kriegserfahrungen im südlichen Bayern 1914-1923, Essen, Klartext, 1997

ZIEMANN, Benjamin, War Experiences in Rural Germany: 1914-1923, Oxford, Berg, 2007.

ZIEMANN, Benjamin, Contested Commemorations. Republican War Veterans and Weimar Political Culture, Cambridge et alii, Cambridge University Press, 2013. 